\title{
Blood Lipids
}

\section{THE ISOLATION OF 3,7,11,15-TETRAMETHYLHEXADECANOIC ACID (PHYTANIC ACID) FROM OX-PLASMA LIPIDS*}

\author{
By A. K. LOUGH \\ Rowett Research Institute, Bucksburn, Aberdeen
}

(Received 11 October 1963)

The presence of an unusual peak on gas-liquid chromatograms of methyl esters of fatty acids derived from ox-plasma lipids was observed by Duncan \& Garton (1963), who noted, in a personal communication to the author, that the "carbon number' (Woodford \& van Gent, 1960) of this component, before and after hydrogenation, was 17.5 on Apiezon $L$ and 16.9 on polymerized ethylene glycol succinate. This chromatographic behaviour resembled that reported by Hawke, Hansen \& Shorland (1959) for the methyl ester of a butterfat component which, though not fully characterized, was apparently a multi-branched $\mathrm{C}_{20}$ fatty acid (Hansen \& Shorland, 1952).

It was therefore considered that the unidentified component of ox-plasma lipids might be a similar branched-chain acid and, on the basis of this supposition, the study now reported was carried out, resulting in the isolation and characterization of the acid as 3,7,11,15-tetramethylhexadecanoic acid, an acid previously prepared chemically from phytol by Willstätter, Mayer \& Huni (1910) and to which they gave the trivial name 'Phytansäure' (i.e. phytanic acid). A preliminary report of the work described in the present paper has been published (Lough, 1963).

\section{MATERIALS AND METHODS}

Fatty acid methyl esters of ox plasma. These were prepared as described by Duncan \& Garton (1963); pooled fatty acid methyl esters from the triglycerides and the phospholipids were taken as the starting material (900 mg.); gas-liquidchromatographic analysis showed the mixed esters to contain $10 \%$ of the unidentified component.

Ketones. Acetone, pentan-2-one, hexan-2-one, heptan-2one, octan-2-one, decan-2-one, undecan-2-one, tridecan-2one and nonadecan-2-one were obtained from $\mathrm{L}$. Light and Co. Ltd., Colnbrook, Bucks. Dodecan-2-one, tetradecan-2one, pentadecan-2-one, hexadecan-2-one, heptadecan-2-one and octadecan-2-one were prepared by the dry distillation of calcium acetate with the calcium salt of the appropriate fatty acid. Each crude ketone was applied, in solution in light petroleum (b.p. $40-60^{\circ}$ ), to a column of silicic acid from which the pure ketone was eluted with $1 \%(v / v)$ ether in light petroleum.

\footnotetext{
* Part 3: Duncan \& Garton (1963).
}

Reference fatty acid methyl esters. Fatty acid methyl esters used as standards in gas-liquid chromatography were prepared from commercially available fatty acids and were purified by fractional distillation in vacuo. The stearic acid used for oxidation with $\mathrm{KMnO}_{4}$ was prepared from chromatographically pure methyl stearate.

Dimethyl ester of 3-methyladipic acid. Methyl hydrogen $\beta$-methylglutarate, prepared from $\beta$-methylglutaric acid (L. Light and Co.) according to the method of Linstead, Lunt \& Weedon (1950), was treated with thionyl chloride (Stallberg-Stenhagen, 1948) to yield the corresponding acid ester chloride. This was allowed to react with diazomethane to give the diazoketone which yielded the dimethyl ester of 3-methyladipic acid on treatment with $\mathrm{Ag}_{2} \mathrm{O}$ in methanol (Bachmann \& Struve, 1942).

Phytanic acid. This was prepared from phytol (L. Light and Co.) either as described by Sonneveld, Begemann, van Beers, Keuning \& Schogt (1962) or according to the method of Karrer, Epprecht \& König (1940), except that in this latter method the acid was isolated by the formation of the potassium soap followed by the removal of non-acidic contaminants by ether extraction and subsequent regeneration of the acid. The $[\alpha]_{500}^{20}$ of the methyl ester was $-0.35^{\circ}$ (c 4 in methanol).

3,7-Dimethyloctanoic acid. Citral (L. Light and Co.) was catalytically hydrogenated by using palladium-charcoal in methanol, and the resulting saturated aldehyde was oxidized with $\mathrm{CrO}_{3}$ and $\mathrm{KHSO}_{4}$, as employed in the preparation of phytanic acid by Karrer et al. (1940) to yield dimethyloctanoic acid.

4,8-Dimethylnonanoic acid. Chain extension of 3,7dimethyloctanoic acid by one carbon atom was effected as described above for 3-methyladipic acid.

Gas-liquid chromatography. Ketones and esters were analysed in an Argon Chromatograph (W. G. Pye and Co. Ltd., Cambridge) by using $4 \mathrm{ft}$. glass columns (internal diam. $4 \mathrm{~mm}$.) at temperatures in the range $50-175^{\circ}$. The stationary phases employed were Apiezon L (Shell Chemicals Ltd., London) and polymerized ethylene glycol succinate prepared in a similar manner to that given by Farquhar, Insull, Rosen, Stoffel \& Ahrens (1959) for the corresponding adipate. Acid-washed Embacel (May and Baker, Ltd., Dagenham, Essex) was used as column support and this was impregnated with $20 \%(w / w)$ polymerized ethylene glycol succinate or $0.7 \%$ (w/w) Apiezon L. Before it was impregnated with Apiezon $L$ the Embacel was treated with dichlorodimethylsilane (British Drug Houses Ltd.) according to the procedure of Silk \& Hahn (1954).

Thin-layer chromatography. Thin layers of Kieselgel G (Merck A.-G., Darmstadt, West Germany) were prepared on glass plates and chromatography was carried out as described by Stahl (1959). 
Infrared spectroscopy. Methyl esters of fatty acids were subjected to infrared analysis as thin films between rocksalt disks by using a twin-beam spectrophotometer model SP. 200 (Unicam Instruments Ltd., Cambridge).

\section{RESULTS}

Treatment of fatty acid methyl esters to concentrate and isolate the unidentified acid. The observation that the ester was relatively less well retained on the polyester phase than on the paraffinic phase ('carbon numbers' 16.9 and 17.5 respectively) indicated the presence of a substituent sufficiently close to the carboxyl group to influence the interaction forces between the ester and the solvent molecules (James, 1959). This possibility prompted an examination for evidence of steric hindrance which is known to be associated with alkyl substitution in the 2- or 3-position relative to the carboxyl group of a branched-chain fatty acid (Smith, 1940; Loening, Garrett \& Newman, 1952). The mixed methyl esters were refluxed for $1.5 \mathrm{hr}$. with a large excess of ethanol containing $1 \%(\mathrm{w} / \mathrm{v})$ of conc. sulphuric acid, and the products of transesterification were subjected to gas-liquid chromatography on Apiezon L. It was found that about half the unknown component was still in the form of its methyl ester, whereas conversion of the other constituents into the corresponding ethyl esters was virtually complete.

The mixed methyl esters (900 mg.) derived from the ox-plasma lipids were treated with mercuric acetate, and the esters of the saturated fatty acids were separated from the mercury adducts of the unsaturated esters by chromatography on silicic acid (Jantzen \& Andreas, 1958). The saturated fatty acid esters $(537 \mathrm{mg}$.) were hydrolysed with ethanolic $0.5 \mathrm{~N}$-potassium hydroxide under reflux for $1.5 \mathrm{hr}$., and the fatty acids were isolated after acidification and ether extraction. The fatty acids were refluxed with methanol containing $1 \%(w / v)$ of conc. sulphuric acid for $1 \mathrm{~min}$., and the product, in ethereal solution, was separated into esters and unesterified fatty acids by extraction with aq. $0.5 \%$ potassium hydroxide. The free fatty acids (132 mg.) were treated with diazomethane and the methyl esters subjected to gas-liquid chromatography on Apiezon L. The unknown ester now constituted about half the total.

Further concentration of the ester was effected by thin-layer chromatography with $3 \%(v / v)$ ether in light petroleum as developing solvent and with a loading of about $10 \mathrm{mg}$. in the form of a narrow band on each of 12 plates (each $20 \mathrm{~cm} . \times 20 \mathrm{~cm}$.). After development of each chromatogram the plate was air-dried and the esters were detected by means of iodine vapour (Malins \& Mangold, 1960). The zone that had migrated in advance of the normal esters was scraped off the plate and, after reduction of the iodine with sulphur dioxide, the adsorbed material was extracted with ether to yield a total of $45 \mathrm{mg}$. Gas-liquid-chromatographic analysis showed the ester to be $\mathbf{9 8 . 5 \%}$ pure; methyl palmitate and methyl stearate were the only 'trace' impurities. The $[\alpha]_{500}^{20}$ of the ester was $-3 \cdot 80^{\circ}$ [c 4 in methanol-chloroform $(2: 1, \mathrm{v} / \mathrm{v})]$.

Infrared-absorption analysis. The infrared-absorption spectrum of the methyl ester of the unknown acid (Fig. 1) suggested considerable discontinuity in the carbon chain since absorption at $720 \mathrm{~cm} .^{-1}$ (associated with a paraffinic chain of four or more methylene groups) was absent; the intense absorption at $1380 \mathrm{~cm} \cdot .^{-1}$ indicated the probable presence of a number of methyl side chains, and a doublet at $1370-1380 \mathrm{~cm} \cdot .^{-1}$ suggested the existence of an isopropyl group (Bellamy, 1958).

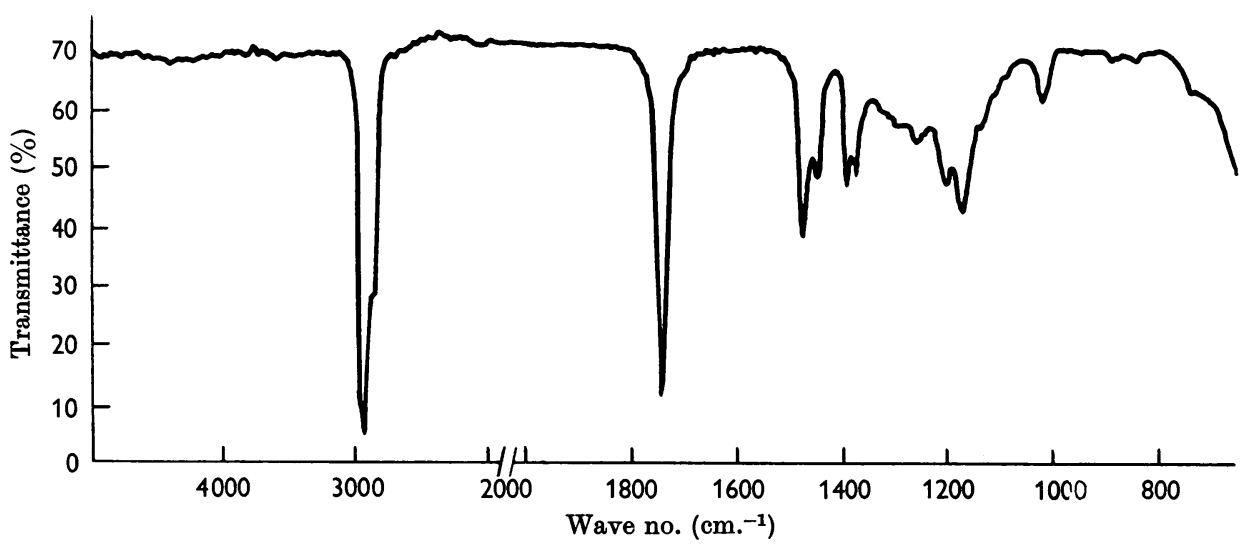

Fig. 1. Infrared-absorption spectrum of the methyl ester of the unknown acid isolated from ox-plasma lipid. 
Oxidative degradation of the isolated acid and its identification as phytanic acid. A sample of the free acid (10 mg.), which was prepared by refluxing the ester with ethanolic $0.5 \mathrm{~N}$-potassium hydroxide for $1.5 \mathrm{hr}$., was treated with potassium permanganate (100 mg.) in acetone (5 ml.) under reflux for $20 \mathrm{hr}$. The acidic and ketonic products were separated as described by Murray (1959) and the acids were converted into methyl esters with diazomethane. Each fraction was then subjected to gas-liquidchromatographic analysis on Apiezon $L$ at several different temperatures to accommodate the wide range of components that were present (Murray, 1959).

In the initial sequence of esters resulting from oxidation of the unknown acid there was no evidence, other than the low yield of one of the components, to indicate the interruption expected from a substituent methyl group presumed to be vicinal to the carboxyl group. However, when a portion of these esters was subjected to transesterification in ethanol, a gas-liquid chromatogram (at $175^{\circ}$ on Apiezon L) of the resultant mixed esters revealed that, of the first six components in descending order of molecular weight, only the third and fourth were apparently free from steric hindrance to the formation of ethyl esters. Since, as noted above, sterically hindered esterification is associated with alkyl branches in the 2- and 3positions relative to the carboxyl group of a fatty acid, it is apparent that the oxidation products could be expected to show the following sequence of components, namely products with substituent groups in the following positions: 3-methyl (i.e. unoxidized starting material); 2-methyl; 5-methyl; 4-methyl; 3-methyl; 2-methyl. This sequence of products can thus be expected from a 3,7-dimethyl fatty acid. Owing to the high temperature at which esters were chromatographed the components of low molecular weight were eluted so rapidly that their identification was not possible. A second portion of the methyl esters was therefore chromatographed on Apiezon L at a comparatively low temperature $\left(105^{\circ}\right)$. This revealed a series of four constituents, a plot of the relative retention volumes of which indicated that they comprised part of an homologous series. The identity of two of the constituents (namely the second and third in order of elution) was established by showing that they were co-chromatographically indistinguishable from the methyl esters of 3,7-dimethyloctanoic acid and 4,8-dimethylnonanoic acid; the other two components were accordingly presumptively identified as the methyl esters of 2,3-dimethylheptanoic acid and 5,9-dimethyldecanoic acid.

It was considered from the foregoing evidence that the unknown acid probably possessed four substituent methyl groups in the form of a saturated isoprenoid chain and that it might well be phytanic acid. Additional evidence in favour of this structure was provided by the chromatograms of the ketone fraction (from the oxidation of the unknown acid with potassium permanganate), in which three main components were present. Relative to the series of normal methyl ketones, these components were found to have "carbon numbers' of $7 \cdot 6,12.0$ and 16.2 , corresponding respectively to methyl ketones with one branch (i.e. an iso-octan-2-one), two branches and three branches. Accordingly authentic phytanic acid (10 mg.) was treated with potassium permanganate (150 mg.) in acetone $(7.5 \mathrm{ml}$.) under reflux for $30 \mathrm{hr}$., and the ketonic fraction was separated from the acidic products as described above. Chromatography of the ketone fraction indicated the presence of three components which were taken to be 2-methylheptan-6-one, 2,6-dimethylundecan-10one and 2,6,10-trimethylpentadecan-14-one. These three components were chromatographically indistinguishable on Apiezon $\mathrm{L}$ from those formed from the unknown acid.

To locate the position of the fourth methyl group an examination was made of the short-chain ketones produced by oxidation of the acid $(3.9 \mathrm{mg}$.) with chromium trioxide (30 mg.) according to the method of Cason, Fessenden \& Agre (1959). After the oxidation mixture was cooled, water $(20 \mathrm{ml}$.) was added followed by powdered potassium disulphite $\left(\mathrm{K}_{2} \mathrm{~S}_{2} \mathrm{O}_{5}\right)$ until reduction of the excess of chromic acid was complete. The solution was divided into two equal parts, one of which was distilled into $10 \mathrm{ml}$. of aqueous dinitrophenylhydrazine reagent (Mann \& Saunders, 1960), the resultant hydrazones being extracted into light petroleum. The other portion was neutralized with aq. $50 \%(\mathrm{w} / \mathrm{v})$ potassium hydroxide and extracted with ether; the ethereal solution, after being washed with water, was added to a methanolic solution of dinitrophenylhydrazine ( $5 \mathrm{ml}$.), prepared according to the method of Mann \& Saunders (1960). After $10 \mathrm{~min}$. the ether was removed in vacuo at room temperature, water $(5 \mathrm{ml}$.) was added and the hydrazones were extracted into light petroleum. Chromatography of the hydrazones on paper according to the methods given by Ellis, Gaddis \& Currie (1958) showed that the only short-chain ketones present were iso-octan-2-one and acetone, thus indicating that the fourth methyl group was attached to the penultimate carbon atom in the fatty acid chain, i.e. a terminal isopropyl group was present in the molecule.

Since oxidation of a branched-chain fatty acid with chromium trioxide in acetic acid is selective with respect to tertiary hydrogen atoms, except where the alkyl substituents are in the 2-, 3- or 4position relative to the carboxyl group (Cason et al. 
1959), 3,7,11,15-tetramethylhexadecanoic acid would be expected to yield, among other products, 3-methyladipic acid, 4,8-dimethylnonanoic acid and 2,6-dimethylundecan-10-one, if somewhat milder conditions of oxidation with chromium trioxide are employed than were used to demonstrate the formation of acetone. Accordingly a further sample of the unknown acid $(2.9 \mathrm{mg}$.) was oxidized with chromium trioxide (6 mg.) in acetic acid $\left(0.3 \mathrm{ml}\right.$.) at $65^{\circ}$ for $3 \mathrm{hr}$. After this oxidation the mixture was refluxed for $1 \mathrm{hr}$. with methanol (5 ml.) contrining $1 \%(\mathrm{w} / \mathrm{v})$ of conc. suphuric acid. After cooling, water $(10 \mathrm{ml}$.) was added and the aqueous methanol extracted with three $10 \mathrm{ml}$. lots of ether. The ether extracts were pooled, washed with water and dried over anhydrous magnesium sulphate; after filtering, the ether was removed under reduced pressure at $0^{\circ}$, to yield a mixture of methyl esters and ketones which were chromatographed on polymerized ethylene glycol succinate. The mixture of methyl esters and ketones, with and without the addition of authentic dimethyl 3methyladipate or authentic methyl 4,8-dimethylnonanoate or the 2,6-dimethylundecan-10-one derived from phytanic acid, was chromatographed on both Apiezon $L$ and polymerized ethylene glycol succinate. It was thus possible to identify 3-methyladipic acid, 4,8-dimethylnonanoic acid and 2,6-dimethylundecan-10-one as being unequivocally present in the oxidation products of the unknown acid.

Gas-liquid chromatography on Apiezon L and polymerized ethylene glycol succinate, at $175^{\circ}$ and $165^{\circ}$ respectively, of a mixture of the methyl ester (isolated from ox-plasma lipids) with methyl phytanate prepared from phytol yielded peaks which were completely coincident. Further, the infrared-absorption spectrum of the synthetic methyl phytanate was identical with that of the methyl ester of the acid isolated from the ox-plasma lipids. It was therefore concluded that the acid was 3,7,11,15-tetramethylhexadecanoic acid.

\section{DISCUSSION}

Influence of branching on the gas-liquid-chromatographic behaviour of fatty acid esters. Gray (1960) showed that the retention volume of the dimethyl acetal of an aliphatic aldehyde compared with that of the methyl ester of the corresponding fatty acid is greater on Apiezon $L$ (non-polar) stationary phase and lower on Reoplex -400 (polar) phase. The present work has shown that methyl phytanate behaves similarly with respect to methyl heptadecanoate in that the phytanate was eluted after methyl heptadecanoate on Apiezon $\mathrm{L}$ and slightly ahead of methyl heptadecanoate on polymerized ethylene glycol succinate. Similarly, Popják \&
Cornforth (1960) provided results from which it can be calculated that methyl 3,7,11-trimethyldodecanoate is eluted after methyl tridecanoate on Apiezon $\mathrm{L}$ and before methyl tridecanoate on polymerized ethylene glycol adipate.

The gas-liquid-chromatographic behaviour of long-chain compounds on polar and non-polar stationary phases is thus influenced by the degree of polarity of functional groups the compounds may contain. Further, the presence of a substituent methyl group at the 3-position of a fatty acid methyl ester has the effect of depressing the degree of polarity of the ester group; thus, whereas the 'carbon number' of methyl phytanate is $\mathbf{1 7 . 5}$ on Apiezon $L$ and 16.9 on polymerized ethylene glycol succinate, the corresponding values for methyl $4,8,12,16$-tetramethylheptadecanoate are 18.5 and 18. 7 respectively (A. K. Lough, unpublished work).

Occurrence and origin of phytanic acid. During the course of the present work Stenhagen (1961) and Sonneveld et al. (1962) reported the occurrence of phytanic acid in ox butterfat; Sonneveld et al. (1962) obtained gas-liquid-chromatographic evidence that accorded well with that of Hansen \& Shorland (1952) for their incompletely-characterized multi-branched $\mathrm{C}_{20}$ fatty acid (also from butterfat) and with that obtained in this Laboratory for the hitherto unidentified fatty acid of ox-plasma lipids. It is thus almost certain that the acid obtained by Hansen \& Shorland (1952) was phytanic acid.

The present study has shown that the unidentified fatty acid found by Duncan \& Garton (1963) in the plasma lipids (particularly the triglycerides and phospholipids) of lactating and non-lactating cows was phytanic acid. It seems reasonable therefore to suppose that phytanic acid present in butterfat is derived from plasma lipids [cf. the conclusion of Riis, Luick \& Kleiber (1960) that both triglycerides and phospholipids of bovine plasma contribute fatty acids for milk glyceride formation]. However, the methyl ester of the acid isolated from butterfat by Sonneveld et al. (1962) had a specific rotation of $+1.2^{\circ}$, whereas the ester prepared in the present investigation from plasma lipids showed a negative rotation that was markedly greater than that of the methyl phytanate prepared from phytol: an explanation for these differences in optical rotation is being sought.

Since farnesoic acid with its branched-chain structure (i.e. 3,7,11-trimethyldodecatrienoic acid) can be formed in the liver (Christophe \& Popjék, 1961), Sonneveld et al. (1962) imply that phytanic acid may have a similar origin. Another possibility is that it may be derived in vivo from phytol present as part of the chlorophyll contained in the animals' feed. Hydrogenation of double bonds is known to be brought about by micro-organisms in 
the rumen (Shorland, Weenink, Johns \& McDonald, 1957), and thus the phytol moiety of ingested chlorophyll could yield dihydrophytol which, if absorbed, would give phytanic acid on oxidation of its primary alcohol group. In this connexion, after the injection of phytol into rabbits Fischer \& Bielig (1940) isolated phytenic acid (i.e. 3,7,11,15tetramethylhexadec-2-enoic acid) from the urine. The hypothesis that phytanic acid can be derived in vivo from feed chlorophyll is being investigated.

\section{SUMMARY}

1. Phytanic acid $(3,7,11,15$-tetramethylhexadecanoic acid) was isolated from ox-plasma lipids.

2. The identity of the acid was established by a study of the products obtained by oxidative degradation and confirmed by showing that it was indistinguishable in respect of its gas-liquid-chromatographic properties and infrared-absorption spectrum from an authentic specimen of the acid.

3. The gas-liquid-chromatographic behaviour of branched-chain acids is briefly discussed, as also is the possibility that plasma phytanic acid may be derived from the phytol moiety of feed chlorophyll.

I thank Dr R. L. M. Synge, F.R.S., for valuable discussion, Dr G. W. Youngson, Robert Gordon's Technical College, Aberdeen, for determining the infrared-absorption spectra, Dr P. M. Scopes and Mr J. P. Jennings of Westfield College, University of London, for determining the optical rotations, and Dr G. A. Garton for his help in the preparation of the manuscript.

\section{REFERENCES}

Bachmann, W. E. \& Struve, W. S. (1942). In Organic Reactions, 3rd ed., Vol. 1, p. 38. Ed. by Adams, R., Bachmann, W. E., Fieser, L. F., Johnson, J. R. \& Snyder, H. R. London: Chapman and Hall Ltd.

Bellamy, L. J. (1958). The Infra-red Spectra of Complex Molecules, 2nd ed. revised. London: Methuen and Co. Ltd.
Cason, J., Fessenden, J. S. \& Agre, C. L. (1959). Tetrahedron, 7, 289.

Christophe, J. \& Popják, G. (1961). J. Lipid Res. 2, 244.

Duncan, W. R. H. \& Garton, G. A. (1963). Biochem. J. 89, 414.

Ellis, R., Gaddis, A. M. \& Currie, C. T. (1958). Analyt. Chem. 30, 475.

Farquhar, J. W., Insull, W., jun., Rosen, P., Stoffel, W. \& Ahrens, E. H., jun. (1959). Nutr. Rev. 17 (suppl.).

Fischer, F. G. \& Bielig, H. J. (1940). Hoppe-Seyl. Z. 266, 73.

Gray, G. M. (1960). J. Chromat. 4, 52.

Hansen, R. P. \& Shorland, F. B. (1952). Biochem. J. 50, 358.

Hawke, J. C., Hansen, R. P. \& Shorland, F. B. (1959). J. Chromat. 2, 547.

James, A. T. (1959). J. Chromat. 2, 552.

Jantzen, E. \& Andreas, H. (1958). Angew. Chem. 70, 656.

Karrer, P., Epprecht, A. \& König, H. (1940). Helv. chim. acta, 23, 272.

Linstead, R. P., Lunt, J. C. \& Weedon, B. C. L. (1950). J. chem. Soc. p. 3331 .

Loening, K., Garrett, A. B. \& Newman, M. S. (1952). J. Amer. chem. Soc. 74, 3929.

Lough, A. K. (1963). Biochem. J. 86, 14 P.

Malins, D. C. \& Mangold, H. K. (1960). J. Amer. Oil Chem. Soc. 37, 576.

Mann, F. G. \& Saunders, B. C. (1960). Practical Organic Chemistry, 4th ed. London, New York and Toronto: Longmans, Green and Co.

Murray, K. E. (1959). Aust. J. Chem. 12, 657.

Popják, G. \& Cornforth, R. H. (1960). J. Chromat. 4, 214. Riis, P. M., Luick, J. R. \& Kleiber, M. (1960). Amer. J. Physiol. 198, 45.

Shorland, F. B., Weenink, R. O., Johns, A. T. \& McDonald, I. R. C. (1957). Biochem. J. 67, 328.

Silk, M. H. \& Hahn, H. H. (1954). Biochem. J. 56, 406.

Smith, H. A. (1940). J. Amer. chem. Soc. 62, 1136.

Sonneveld, W., Begemann, P. H., van Beers, G. J., Keuning, R. \& Schogt, J. C. M. (1962). J. Lipid Res. 3, 351.

Stahl, E. (1959). Pharm. Rdsch. 2, 1.

Stallberg-Stenhagen, S. (1948). Ark. Kemi Min. Geol. 26, 1.

Stenhagen, E. (1961). Z. anal. Chem. 181, 462.

Willstätter, R., Mayer, E. W. \& Huni, E. (1910). Liebigs Ann. 378, 73.

Woodford, R. P. \& van Gent, C. M. (1960). J. Lipid Res. $1,188$. 